\title{
Jean-Charles BONENFANT
}

Journaliste et bibliothécaire québécois (1912-1977)

Professeur de science politique, Université Laval.

(1962)

\section{"Les études politiques.”}

Un document produit en version numérique par Diane Brunet, bénévole,

Diane Brunet, bénévole, guide, Musée de La Pulperie, Chicoutimi

Courriel: Brunet_diane@hotmail.com

Page web dans Les Classiques des sciences sociales

Dans le cadre de: "Les classiques des sciences sociales"

Une bibliothèque numérique fondée et dirigée par Jean-Marie Tremblay, professeur de sociologie au Cégep de Chicoutimi

Site web: http://classiques.uqac.ca/

Une collection développée en collaboration avec la Bibliothèque

Paul-Émile-Boulet de l'Université du Québec à Chicoutimi

Site web: http://bibliotheque.uqac.ca/ 


\section{Politique d'utilisation de la bibliothèque des Classiques}

Toute reproduction et rediffusion de nos fichiers est interdite, même avec la mention de leur provenance, sans l'autorisation formelle, écrite, du fondateur des Classiques des sciences sociales, Jean-Marie Tremblay, sociologue.

Les fichiers des Classiques des sciences sociales ne peuvent sans autorisation formelle:

- être hébergés (en fichier ou page web, en totalité ou en partie) sur un serveur autre que celui des Classiques.

- servir de base de travail à un autre fichier modifié ensuite par tout autre moyen (couleur, police, mise en page, extraits, support, etc...),

Les fichiers (.html, .doc, .pdf, .rtf, .jpg, .gif) disponibles sur le site Les Classiques des sciences sociales sont la propriété des Classiques des sciences sociales, un organisme à but non lucratif composé exclusivement de bénévoles.

Ils sont disponibles pour une utilisation intellectuelle et personnelle et, en aucun cas, commerciale. Toute utilisation à des fins commerciales des fichiers sur ce site est strictement interdite et toute rediffusion est également strictement interdite.

L'accès à notre travail est libre et gratuit à tous les utilisateurs. C'est notre mission.

Jean-Marie Tremblay, sociologue

Fondateur et Président-directeur général, LES CLASSIQUES DES SCIENCES SOCIALES. 
Cette édition électronique a été réalisée par Diane Brunet, bénévole, guide, Musée de La Pulperie, Chicoutimi à partir du livre de :

à partir du texte de:

Jean-Charles Bonenfant

\section{“Les études politiques.”}

Un texte publié dans l'ouvrage sous la direction de Fernand Dumont et Yves Martin, Situation de la recherche sur le Canada français, pp. 75-82. Premier colloque de la revue RECHERCHES SOCIOGRAPHIQUES du département de sociologie et d'anthropologie de l'Université Laval. Québec: Les Presses de l’Université Laval, 1962, 296 pp.

[Autorisation formelle confirmée le 6 février 2006 au téléphone par M. Yves Martin et confirmée par écrit le 7 février 2006 de diffuser la totalité de ses œuvres: articles et livres]

Courriel : yves_martin@sympatico.ca

Polices de caractères utilisée :

Pour le texte: Times New Roman, 14 points.

Pour les citations : Times New Roman 12 points.

Pour les notes de bas de page : Times New Roman, 12 points.

Édition électronique réalisée avec le traitement de textes Microsoft Word 2008 pour Macintosh.

Mise en page sur papier format : LETTRE US, 8.5’’ x 11’’.

Édition numérique réalisée le 5 avril 2013 à Chicoutimi, Ville de Saguenay, province de Québec, Canada. 


\section{Jean-Charles Bonenfant}

Département de science politique, Université Laval

Bibliothèque de la Législature, Québec

\section{"Les études politiques.”}

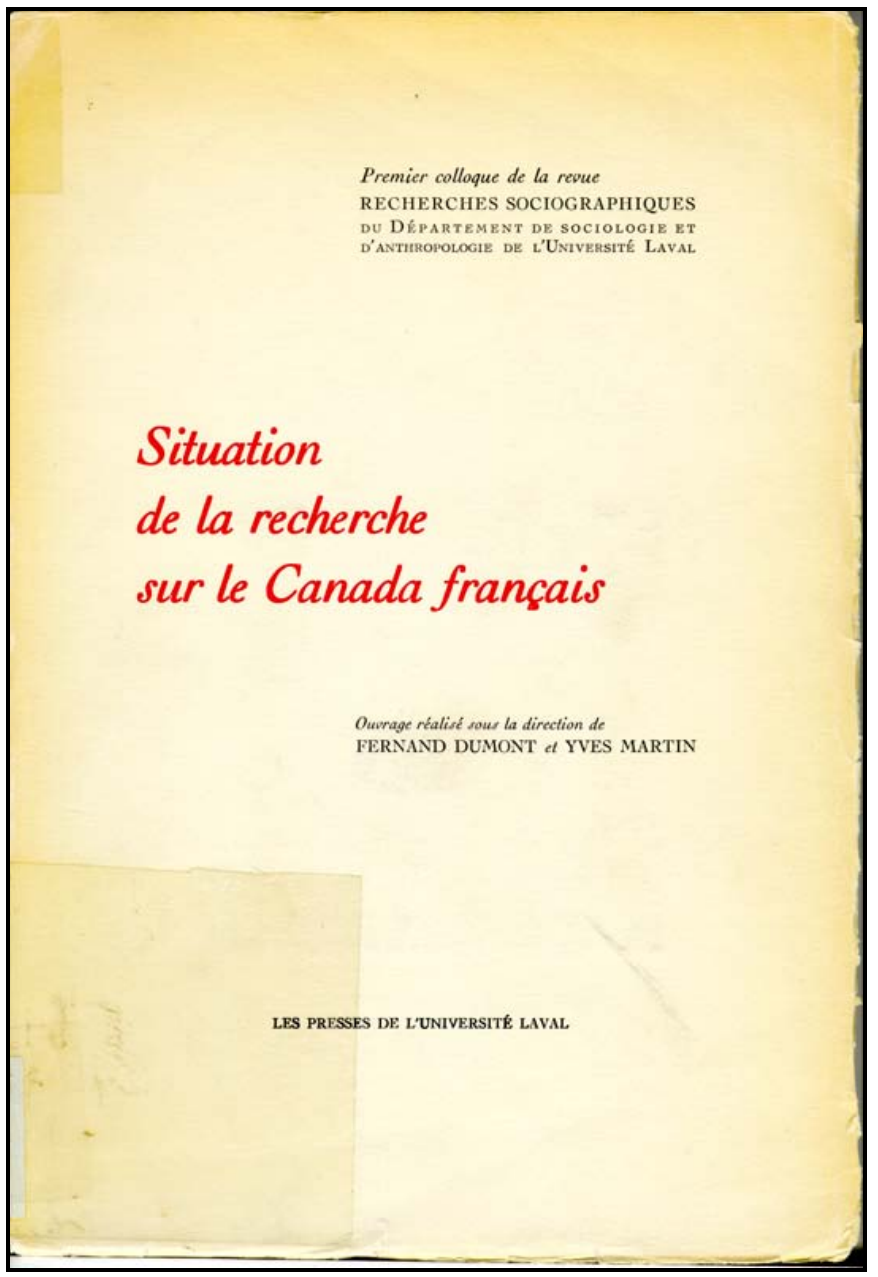

Un texte publié dans l'ouvrage sous la direction de Fernand Dumont et Yves Martin, Situation de la recherche sur le Canada français, pp. 75-82. Premier colloque de la revue RECHERCHES SOCIOGRAPHIQUES du département de sociologie et d'anthropologie de l'Université Laval. Québec: Les Presses de l’Université Laval, 1962, 296 pp. 


\title{
Situation de la recherche sur le Canada français I. Perspectives historiques
}

\section{Jean-Charles Bonenfant}

Département de science politique, Université Laval

Bibliothèque de la Législature, Québec

\section{"Les études politiques."}

\author{
[pp. 75-82.]
}

\section{$\underline{\text { Retour à la table des matières }}$}

Science de carrefour et science de synthèse, dont l'objet est l'autorité, les gouvernants et le pouvoir, la science politique peut être tentée par l'impérialisme. Aussi, pour bien en délimiter les frontières et pour ne pas empiéter sur le domaine d'autrui, j'ai cru devoir adopter comme rubriques fondamentales de mon étude, celles qu'ont précisées un groupe d'experts réunis par l'Unesco à Paris, en 1948, soit : la théorie politique, les institutions politiques, les partis, les groupes et l'opinion publique, et finalement les relations internationales. ${ }^{1}$ À la demande des organisateurs du colloque, j'ai ajouté à ces rubriques le droit. Ce n'est pas une hérésie complète puisque, à la rigueur, une bonne partie du droit peut être incluse dans les institutions politiques ; c'est en mê-

1 La science politique contemporaine. Contribution à la recherche, la méthode et l'enseignement, Unesco, 1950, 4. 
me temps pour la science politique une douce vengeance vu que, jusqu'ici, dans notre province surtout, elle s'est trop souvent confondue avec le droit constitutionnel.

Lorsqu'il y a une quinzaine d'années, Burton G. Keirstead et Frederick M. Watkins préparèrent pour l'Unesco un bilan de la science politique au Canada, ils ne purent citer qu'un seul nom canadien-français, celui de Henri Bourassa comme représentant de ce qu'ils appelaient « quelques pamphlétaires et quelques journalistes inspirés » dont, ajoutaient-ils « on ne saurait ranger (les) écrits passionnés parmi les ouvrages scientifiques $» .{ }^{2}$ Les deux universitaires canadiens-anglais ne furent pas injustes à notre égard, car nous verrons que le bilan de notre science politique est plutôt mince.

La première rubrique de l'Unesco est donc la théorie politique à laquelle on a joint l'histoire des idées politiques. C'est un double domaine qu'on n'a guère abordé au Canada français. $\mathrm{Si}$, pour utiliser les mots de Jean Meynaud, 3 « la théorie forme un ensemble articulé de définitions, d'assomptions et de propositions couvrant un sujet déterminé » et "doit permettre de présenter des relations susceptibles de vérification », elle n'a [76] pas beaucoup été pratiquée ici. Lorsqu'on s'est intéressé à des phénomènes comme le nationalisme, le fédéralisme, le bicaméralisme, on l'a fait dans le concret, le plus souvent même dans une atmosphère de polémique. Il y a tout de même de la théorie politique dans ce livre de précurseur que publia à Paris, en 1896, Edmond de Nevers, sous le titre de L'avenir du peuple canadienfrançais ; on en trouve dispersée dans les écrits de l'abbé Wilfrid Morin, de M Esdras Minville, du R. P. Richard Arès et dans les livraisons de l'Action française de 1918 à 1928 et de l'Action nationale de 1933 à nos jours. Le plus loin où l'on ait osé se rendre en ce domaine est peut-être contenu dans le volume II du Rapport de la Commission royale d'enquête sur les problèmes constitutionnels où on retrouve, malgré l'anonymat de la rédaction, les principales idées de M. Minville et du R. P. Arès sur l'État et sur le fédéralisme.

2 Ibid., 178.

3 Jean MEYNAUD, Introduction à la science politique, Paris, Librairie Armand Colin; 1959, 18 (Cahiers de la Fondation nationale des sciences politiques, 100). [Livre disponible dans Les Classiques des sciences sociales. JMT.] 
Quant à l'histoire des idées politiques, elle n'a même pas été esquissée. Nous ne possédons qu'une chronique des événements dans les trente-trois tomes de l'Histoire de la province de Québec, de Robert Rumilly, et une synthèse plus concise avec quelques jugements de valeur dans The French Canadians 1760-1945, de Mason Wade.

Au chapitre des institutions politiques, qui comprend d'abord l'étude de la constitution et celle des relations entre le gouvernement central et les gouvernements locaux, nous avons été un peu plus féconds pour autant que les problèmes étaient envisagés sous un éclairage juridique. Les Pères de la Confédération, que ce soient les quatre Canadiens français ou leurs vingt-neuf collègues canadiens-anglais, n'étaient pas de profonds penseurs ; ils ne citaient pas, comme leurs prédécesseurs américains de 1787, Locke et Montesquieu. Ce n'étaient que des praticiens de la politique à la recherche de solutions immédiates et on leur a prêté, au Canada français surtout, de belles idées auxquelles ils n'ont jamais songé. Aussi, faut-il attendre à 1883 et 1884 pour que soient publiées trois lettres du juge T.-J.-J. Loranger sur L'interprétation de la constitution fédérale, lettres qui constituent le premier commentaire canadien-français ayant quelque valeur sur l'Acte de l'Amérique du Nord britannique. En 1889, le futur juge P.-B. Mignault publiait son Manuel de droit parlementaire dont le second titre Cours élémentaire de droit constitutionnel reflète davantage le contenu qui n'est pas sans une certaine valeur. Et les années passèrent sans que s'enrichisse substantiellement notre bibliothèque constitutionnelle jusqu'au moment où la menace que le Statut de Westminster permette au pouvoir central d'être entièrement maître de l'Acte de l'Amérique britannique du Nord et de ses modifications vienne en quelque sorte magnifier la théorie du pacte. Cette théorie fut énoncée officiellement en septembre 1930 par le premier ministre d'Ontario, G. H. Ferguson, mais c'est surtout dans le Québec qu'elle fut ensuite utilisée. Si on examine les nombreuses références que le R. P. Arès a recueillies à ce sujet, soit dans Dossier sur le pacte fédératif publié en 1941 [77] ou dans La Confédération, pacte ou loi ? publié quelques années plus tard, on s'aperçoit que, malheureusement, nos constitutionalistes manquèrent d'imagination ou se montrèrent paresseux, se contentant le plus souvent d'invoquer en faveur de la théorie du pacte des arguments d'autorité. D'ailleurs, à propos de la nature de notre constitution formelle, nous avons manqué une belle occasion de nous débarrasser 
de notre conception primitive, presque charismatique du droit et le plus souvent stérile en n'approfondissant pas davantage la théorie de l'institution. Quelques Canadiens français l'esquissèrent, comme Léopold Richer dans Notre problème politique ${ }^{4}$ et Edouard Laurent dans un des premiers Cahiers de l'École des sciences sociales, politiques et économiques de Laval, intitulé Quelle est la nature de l'Acte de 1867 ? Le R.P. J.-T. Delos, grand spécialiste de la question, en causa avec professeurs et élèves pendant son séjour au Canada, mais les praticiens même les plus évolués, comme $\mathrm{M}^{\mathrm{e}}$ Antonio Perrault, se scandalisèrent de ces intrus qui venaient ébranler les cadres traditionnels du droit. 5

Lorsque fut créée par Ottawa, en 1937, la Commission royale des relations entre le Dominion et les provinces, événement décisif dans le développement de la science politique au Canada, nous n'avions guère étudié notre constitution et nous étions en ce domaine en retard sur les Canadiens anglais. Chez eux, selon le témoignage de Kirstead et Watkins, « la science politique... (était) demeurée dans une large mesure une branche subalterne de la science économique ». ${ }^{6}$ Mais ils avaient tout de même eu un Stephen Leacock, qui fut autre chose qu'un humoriste, et un O.D. Skelton dont l'essai sur le socialisme avait été jugé digne de figurer dans les quelque cinquante livres qu'on plaça dans le tombeau de Lénine. Les Canadiens anglais possédaient surtout un nombre considérable d'universitaires qui purent participer au travail de la Commission Rowell-Sirois. Les Canadiens français n'y furent représentés momentanément que par le juge Thibaudeau Rinfret, qui était un civiliste, et ensuite par un notaire d'ailleurs intelligent et sympathique mais qui n'enseignait que par à côté le droit constitutionnel à Laval.

Parmi les auteurs des nombreuses études qui furent préparées pour la Commission, on ne trouve que deux noms canadiens-français, celui de $\mathrm{M}$. Esdras Minville et celui de $\mathrm{M}^{\mathrm{e}}$ Léon Mercier-Gouin. En résumé, nous ne pouvions offrir à la Commission un Robert Alexander Mac-

4 Léopold RICHER, Noire problème politique, Montréal, Éditions de l'A.CF., 1938, 122 et suiv.

5 Revue du Barreau, 1942, 355-359.

6 La science politique contemporaine. Contribution à la recherche, la méthode et l'enseignement, Unesco, 1950, 178. 
Kay, professeur de science politique à l'Université Dalhousie, un Henry Forbes Angus, professeur d'économie politique à l'Université de la Colombie britannique, et un grand journaliste politique comme John W. Dafoe, qui, en définitive, [78] préparèrent le Rapport de la Commission et orientèrent ainsi pour longtemps le fédéralisme canadien.

C'est tout de même jusqu'à un certain point des idées énoncées par la Commission Rowell-Sirois que devait naître, en 1954, un de nos premiers livres de science politique véritable. Le fédéralisme canadien, de Maurice Lamontagne. Enfin, au Canada français, on abordait l'étude du fédéralisme en dehors des strictes œillères juridiques. Certes, M. Lamontagne s'est fait des adversaires pour bien d'autres raisons, mais inconsciemment ceux qui le combattirent le firent parce qu'ils sentaient que le fédéralisme échappait à leur étude exclusive et pouvait être examiné en tenant compte d'éléments autres que les textes sacro-saints des lois ou des décisions du Comité judiciaire du Conseil privé.

Puis ce fut, en 1953, la création d'une autre commission, québécoise cette fois, la Commission royale d'enquête sur les problèmes constitutionnels, la commission Tremblay. Quelques-uns des mémoires qui lui furent soumis, son rapport publié en 1956 et au moins une des annexes à ce rapport, celle de M. François-Albert Angers, sur La centralisation et les relations fédérales-provinciales, ont contribué à l'enrichissement de notre science politique. Je note aussi que les commissaires, ayant sans doute souffert eux-mêmes de l'absence de tant de monographies qui leur auraient été utiles, recommandèrent à l'Etat provincial de favoriser dans toute la mesure du possible l'organisation de la recherche dans les universités et les centres d'enseignement supérieur dans toutes les branches du savoir et particulièrement dans certaines disciplines qu'ils énumèrent, dont « la science politique ». ${ }^{7}$

Il est d'ailleurs intéressant de constater que ce sont peut-être jusqu'ici les enquêtes des commissions royales qui, dans le Québec, ont fait accomplir le plus de progrès à la science politique si bien qu'en 1957, M. Gérard Bergeron a pu intituler Problèmes politiques du Québec un répertoire bibliographique des commissions royales d'en-

7 Rapport de la Commission royale d'enquête sur les problèmes constitutionnels, vol. III, tome II, 324. 
quête, présentant un intérêt spécial pour la province de Québec de 1940 à 1957.

Sous la rubrique des institutions politiques, la classification de l'Unesco inclut l'administration publique. En cette matière, nous n'avons rien produit. Nos hommes politiques ont beaucoup disserté sur les bienfaits ou les dangers du développement de l'action de l'État, mais jamais nous n'avons étudié scientifiquement ce qui constitue, à mon sens, un des problèmes les plus aigus de la science politique moderne, c'est-à-dire le contrôle de l'acte administratif, la façon de protéger l'individu en face d'un État qui devient nécessairement de plus en plus puissant.

La troisième rubrique de l'Unesco traite des partis, des groupes et de l'opinion publique. C'est un domaine passionnant de la science politique où elle ne se distingue pas toujours très bien de la sociologie «si d'ailleurs, [79]comme dirait malicieusement Duverger, elles se distinguent autrement que par les départements ". ${ }^{8}$ C'est un domaine qui s'est développé considérablement en France et en Angleterre ces dernières années et c'est un domaine dans lequel nous commençons à balbutier. L'histoire de nos partis politiques n'a pas été écrite ; nous avons un peu pratiqué la sociologie électorale et, récemment, les partis politiques semblent avoir compris qu'il $y$ avait pour eux-mêmes un intérêt immédiat à sonder scientifiquement l'opinion publique. Je signale en particulier Les électeurs québécois, Attitudes et opinions à la veille de l'élection de 1960, rapport d'un groupe de recherches sociales, de Montréal, derrière lequel agissait le parti libéral. Je n'oublie pas non plus l'intéressante étude de Jean Hamelin, Jacques Letarte et Marcel Hamelin, publiée en 1960, dans les Cahiers de géographie de Québec, dont les auteurs eux-mêmes ont admis que ce n'était pas un essai de science politique mais uniquement un instrument de travail.

Enfin, sous la quatrième rubrique de l'Unesco, relations internationales, les travaux canadiens-français ont été plutôt rares car pendant longtemps il fut même élégant de se moquer des organismes internationaux.

8 Maurice DuVERGER, dans le Traité de sociologie, publié sous la direction de Georges Gurvitch, Presses Universitaires de France, tome second, 1960, 3. 
On me reprochera peut-être de ne pas avoir dressé un inventaire méthodique et complet de nos richesses. C'était impossible et j'en ferai d'ailleurs, dans un instant, l'objet d'une recommandation. Mais même si on peut me citer quelques ouvrages oubliés, je pense que nous admettons tous notre pauvreté en science politique. Pour nous en convaincre par des arguments indirects, je vous invite à quelques petites expériences de recherche auxquelles par besoin vous vous êtes peut-être d'ailleurs livrés. Ouvrez par exemple la bibliographie que le gouvernement canadien a publiée en 1951 des Thèses des gradués canadiens dans les humanités et les sciences sociales de 1921 à 1946 ; parcourez les chapitres consacrés à la science politique et aux sciences connexes et vous verrez que l'apport canadien-français est assez maigre. Prenez les deux index décennaux du Canadian Journal of Economies and Political Science qui couvrent une période allant de 1935 à 1954 et qui permettent de connaître directement ou indirectement, par la bibliographie, tout ce qui s'est publié de valable en science politique au Canada depuis environ un quart de siècle et vous constaterez que nous n'avons pas fourni grand-chose. Enfin, lorsque en science politique nous voulons pratiquer l'humilité, nous songeons à toutes les belles études qui ont été publiées dans la série Canadian Government par University of Toronto Press, études parmi lesquelles nous pouvons au moins nous enorgueillir de l'ouvrage de M. Paul Gérin-Lajoie sur les amendements à la constitution. Nous pensons aussi aux quelque dix ouvrages consacrés à l'arrière-plan et au développement du crédit social en Alberta. Et nous pensons à tout ce que nous pourrions faire !

[80]

Il me reste à dire un mot du droit. Dieu sait si nous l'avons pratiqué. Nous avons produit ce que nous appelons de savants juges et d'habiles avocats qui se sont contentés de faire de l'interprétation et qui, très rarement, ont témoigné d'imagination juridique. Trois traités généraux de droit civil ont été publiés, celui de Langelier, celui de Mignault et celui qui se termine actuellement chez Wilson \& Lafleur. Ce sont trois ouvrages qui, en France, seraient classés parmi ceux de l'école classique dite de l'exégèse, une école qui, depuis la fin du siècle dernier, a été remplacée par l'école dite moderne, celle dont les tenants, pour employer les termes du doyen Léon Julliot de la Morandière, " ne se contentent plus du commentaire logique de la loi » mais « font intervenir dans leur exposé et leur critique des solutions légales, 
des considérations économiques, sociales » et "se préoccupent du droit comparé, des exemples donnés par les législations étrangères et surtout prennent conscience du rôle de la coutume, de la place essentielle de la jurisprudence $» .9$

À vrai dire, nous n'avons qu'un ouvrage général de ce genre. C'est la synthèse que le professeur Louis Baudoin, de McGill, a publiée en 1953, sous le titre Le droit civil de la province de Québec, modèle vivant de droit comparé.

Au lendemain de 1930, nous eûmes tout de même une polémique juridique qui opposa les causalistes et les anti-causalistes. En 1936, $\mathrm{M}^{\mathrm{e}}$ Marcel Faribault eut l'originalité d'appliquer la théorie de l'institution à la fiducie dans un livre, intitulé Traité théorique et pratique de la fiducie ou trust du droit civil dans la province de Québec, qui fut cité à l'étranger. En 1941, fut fondée la Revue du Barreau, dans laquelle $\mathrm{M}^{\mathrm{e}}$ Antonio Perrault et après lui quelques praticiens osèrent critiquer les arrêts de tribunaux. En 1934, 1939, 1952 et 1958, les réunions au Canada français de juristes du monde entier groupés dans l'Association Henri-Capitant donnèrent à quelques avocats et notaires la tentation de voir le droit en dehors des dossiers de leurs cabinets. Enfin, ces dernières années, une génération de jeunes professeurs est née dans nos Facultés de droit et nous offre maintenant des ouvrages originaux que je n'ai pas le temps d'énumérer ici, mais dont le livre de $\mathrm{M}^{\mathrm{e}}$ André Morel, Les limites de la liberté testamentaire dans le droit civil de la province de Québec, publié à Paris en 1960, me semble le plus bel exemple. Il reste que notre droit s'est développé au hasard de la jurisprudence et des amendements des parlements et qu'il est terriblement en retard sur les faits.

Le bilan que je viens d'esquisser est assez pauvre en science politique comme en droit, mais les chercheurs ne peuvent qu'en être heureux car ils éprouvent une joie professionnelle à constater que leur champ de travail n'est pas encore défriché. Mais il y a tant à faire qu'il faut, surtout [81] dans une petite société comme la nôtre où le nombre des travailleurs sérieux demeurera longtemps restreint, l'accomplir avec méthode et coopération.

9 Léon JULLIOT DE LA MORANDiÈRE, Introduction à l'étude du droit civil français, Introduction à l'étude du droit, Paris, 1951, 241. 
Pour éviter de répéter inutilement le travail déjà accompli et pour utiliser tous les matériaux disponibles, il faut d'abord dresser l'inventaire complet, définitif et analytique des travaux et des documents. Il est urgent que nous ayons pour les publications gouvernementales du Québec de 1867 à nos jours une bibliographie analogue à celles qui ont été soigneusement rédigées pour les provinces des Prairies, la Colombie britannique et le Nouveau-Brunswick. Souhaitons aussi que le gouvernement du Québec multiplie ses publications et les mette à la disposition de tous. Ottawa nous offre depuis une quinzaine d'années un excellent service de documents variés. À Québec, c'est la pauvreté et le désordre parfait. Pour ma part, avec toute la prudence d'un fonctionnaire, je réclamais déjà en 1956 dans une communication devant la Société canadienne d'éducation des adultes un meilleur système de distribution des publications du Québec. Je pense que c est toujours urgent. Si le rapport de la Commission Tremblay a été jadis aussi difficile à obtenir qu'un exemplaire de l'Amant de Lady Chatterley avant le jugement de la Cour suprême, ce n'est pas surtout parce que le premier ministre de l'époque en redoutait inconsciemment le contenu, mais c'est parce qu'il n'existait pour une telle publication aucun système normal de distribution. Si, depuis deux ans, vous avez eu besoin de quelques publications qui, à mon sens, sont indispensables aux politicologues du Québec, voici, si vous aviez la chance d'être bien renseignés, ce que vous avez dû faire. Pour obtenir le Rapport des élections de 1960, vous avez écrit au Président général des élections ; pour obtenir L'Annuaire statistique de la Province, vous vous êtes adressés au directeur du Bureau des statistiques ; c'est au bureau du Premier ministre qu'on distribuait L'Étude préliminaire à la révision de la carte électorale de la province de Québec et c'est au bureau de la distribution de l'Assemblée législative que vous pouviez obtenir le Bill 15, la Loi électorale de Québec. Et je pourrais pendant longtemps continuer la promenade.

Nous avons aussi besoin de plus de coopération entre les Universités et même entre les Facultés, les instituts, et il faut songer à une distribution du travail entre tous ceux qui, au Canada, s'intéressent à la science politique et au droit. Au niveau de l'enseignement supérieur, notre petite société ne peut se donner le luxe de la concurrence. Nous pouvons même souhaiter qu'un certain dirigisme permette d'orchestrer 
le travail de recherche, d'éviter la duplication et d'orienter les étudiants vers les monographies dont nous avons le plus besoin.

Enfin, il nous faut surtout des professeurs, pas des improvisateurs autodidactes, comme ceux de ma génération, mais des professeurs auxquels je souhaite avec envie une formation canadienne, américaine, britannique [82] et française, car notre science politique et notre droit doivent être plus qu'ailleurs le fruit d'une synthèse.

Ne croyons pas toutefois que le développement de la science politique transformera radicalement notre société. Comme l'a écrit Jean Meynaud, même si l'on consacrait à la science politique des sommes égales à celles affectées à la physique nucléaire ou à la chimie, on n'arriverait pas à des résultats de même portée et il en découle que « la modestie demeurera longtemps l'une des vertus les plus nécessaires au spécialiste en science politique. » 10

Tout de même, dans une société où l'on est porté à croire que les changements s'opèrent surtout par les hommes, il est peut-être temps que l'on songe à modifier les institutions, et pour cela la connaissance et l'utilisation des données de la science politique sont évidemment nécessaires.

Jean-Charles Bonenfant

Département de science politique, Université Laval ;

Bibliothèque de la Législature, Québec. 


\title{
COMMENTAIRE
}

\author{
Vincent Lemieux \\ Département de science politique, \\ Université Laval.
}

\section{[pp. 83-85.]}

$\underline{\text { Retour à la table des matières }}$

Je ne m'emploierai pas ici à relever les études oubliées, volontairement ou non, par $\mathrm{M}$. Bonenfant. Ou plutôt je n'en relèverai qu'une : Les institutions politiques canadiennes 11 de qui vous savez. M. Bonenfant m'en voudra peut-être, mais je soutiendrai quand même qu'en attendant l'ouvrage complet qu'il nous prépare, ce petit volume d'entretiens doit être intégré à l'inventaire de nos quelques produits originaux.

Ceci dit, je me limiterai, durant les quelques minutes qui me sont allouées, à l'une des quatre rubriques posées par les experts de l'Unesco, celle des partis, des groupes politiques et des opinions publiques. Certains travaux déjà faits ou entrepris, mais qui sont encore peu connus, retiendront mon attention, et aussi certains travaux à faire, dont vous vous doutez bien qu'ils sont nombreux. À la fin, je serai amené à déborder sur le secteur des institutions, ainsi que sur celui de la théorie et de l'histoire des idées, ce qui montrera, si besoin en est, que le développement dans un secteur des sciences politiques ne pourra que mettre en question, puis en branle, d'autres secteurs.

11 Jean-Charles Bonenfant, Les institutions politiques canadiennes, Québec, Les Presses Universitaires Laval, 1954, 204 p. 
Comme vient de le dire $\mathrm{M}$. Bonenfant, nous avons un peu pratiqué la sociologie électorale. En fait, nous commençons de la pratiquer. À côté de l'étude de Jean et de Marcel Hamelin et de Jacques Letarte, il faut mettre les thèses inédites de Paul Cliché et de Harold Angell, restreintes la première à la période 1927-1956, la seconde aux élections provinciales des années vingt, qui, par le débrouillage qu'elles ont accompli devraient permettre des travaux plus poussés. Au niveau des circonscriptions électorales, nous possédons aussi quelques essais de mise en ordre. La première étude a porté sur la circonscription de Lévis, une autre porte actuellement sur celle de Chicoutimi, et des calculs statistiques sont disponibles sur les circonscriptions de Beauce, de Portneuf et de Saint-Maurice. Toutefois, il n'existe pas, à ma connaissance, de travaux comparables sur les élections fédérales. Quant aux quelques recherches entreprises sur les élections et référendums municipaux, elles ont surtout montré des antagonismes internes aux municipalités : antagonismes entre quartiers à Sherbrooke, antagonismes entre vieux résidents et « immigrés » à Saint-Romuald.

Des premiers travaux portant sur les circonscriptions provinciales, certaines constantes se dégagent. Ainsi, les traditions électorales des localités composant une circonscription sont très diverses, mais les variations du vote d'une élection à l'autre sont beaucoup moins originales : si, par exemple, l'Union Nationale en reprend dans l'ensemble de la circonscription, elle en reprend aussi généralement dans à peu près toutes les localités, bien loin que de forts gains dans les localités «bleues » viennent effacer de moins fortes pertes dans les localités « rouges ». Un autre phénomène remarquable, qui se produit fréquemment dans Portneuf, est l'opposition dans une même localité entre le village et la paroisse, entendez par celle-ci le milieu plus proprement rural. C'est ainsi qu'une forte majorité « rouge » dans le village peut s'accompagner d'une non moins forte majorité «bleue » dans la paroisse, et vice versa. Je signalerai enfin un autre phénomène assez étonnant, que j'ai remarqué dans Lévis, phénomène d'ailleurs disparu : soit l'appui quasi unanime que donnaient des petites localités à l'un ou l'autre parti.

[84]

Donc, au niveau de la province et des circonscriptions, l'enquête est commencée. Au niveau des individus aussi, où le rapport du Groupe de recherches sociales, sur les opinions et attitudes des électeurs 
québécois à la veille des élections de 1960, a proposé de premières hypothèses. À Montréal, des étudiants de science politique ont entrepris une recherche sur le militantisme politique de certains partisans libéraux. Mais entre les deux niveaux, c'est l'inconnu. Je suis pourtant convaincu, confirmé en cela par la lecture des meilleurs travaux français, anglais et américains, que c'est au niveau des localités, des petites collectivités politiques, qu'il y a le mieux à faire.

Des phénomènes politiques municipaux ont été étudiés à Beauport, à Lévis, à Sherbrooke, mais que savons-nous, par exemple, des connexions entre affiliations politiques et affiliations de parenté ? Pas beaucoup plus que ce qui se trouve dans Miner. Sur cette question précise, tout est à faire, car si nos sociologues ont beaucoup discuté de la famille canadienne-française, ils ne nous ont donné que quelques études superficielles des réseaux de parenté et des attitudes qui les sous-tendent. Personne n'a suffisamment montré comment la parenté chez nous s'est instituée contre la société politique et sa coordination propre. Nous ne savons scientifiquement rien de l'organisation provinciale et locale de nos partis politiques, des techniques qu'ils emploient. Tout au plus un premier inventaire et une première interprétation de leur propagande ont-ils été tentés récemment par un étudiant.

Pourtant nous ne pourrons pas prétendre à une sociologie un peu rigoureuse du patronage politique, sociologie qui viendrait à son heure, tant que ces faits d'affiliation, d'organisation et de propagande partisanes n'auront pas été éclairés. Et comme c'est lors d'une campagne électorale que ces faits se manifestent le mieux, il faudrait aller les saisir à ce moment. Pourquoi ne pourrait-on pas considérer ces campagnes comme un composé de potlach et d'interrègne, une espèce de phénomène politique total où sont renouvelées les alliances et réévalués les prestiges, où de fortes sommes sont dépensées en quelques jours, où des prestations et contre-prestations sont échangées : votes contre promesses, quand ce n'est pas contre boissons fortes ou appareils de télévisions ? Lisez avec cette hypothèse en tète Les mœurs électorales dans le Québec de Jean et Marcel Hamelin, et vous verrez apparaître la dimension sociale du problème, trop souvent ignorée sous la dimension morale.

Des candidats à ces élections, des députés élus, que savons-nous ? Voilà un autre champ de sociologie politique qui est bien peu développé et pas seulement chez nous. Pourtant une brève enquête, faite 
par un de mes étudiants, révèle un fait aussi étonnant que celui-ci : de 1867 à aujourd'hui, 10\% des députés fédéraux nouvellement élus dans l'Ontario ont moins de 35 ans, mais dans le Québec cette proportion est de 22\%. Pourquoi ? Dans une certaine mesure parce que les nouveaux députés libéraux sont en moyenne plus jeunes que les nouveaux députés conservateurs, et que ces nouveaux députés libéraux sont proportionnellement plus nombreux dans le Québec que dans l'Ontario ; mais, sans doute, d'autres facteurs jouent aussi. Voilà une piste pleine de promesses pour qui voudrait évaluer notre apport à la Confédération canadienne.

M. Bonenfant remarquait avec raison que nous n'avons rien produit en science de l'administration publique. Je signalerai toutefois qu'une enquête est en cours sur certaines caractéristiques de quelque 200 hauts fonctionnaires de la province de Québec, sans qu'on puisse encore voir ce qui [85] en sortira. Non seulement nous n'avons jamais étudié scientifiquement le problème du contrôle de l'acte administratif, mais l'histoire de nos différents ministères, leur organisation, leur fonctionnement n'ont pas encore tenté les chercheurs. C'est d'autant plus regrettable que ceux-ci disposent aujourd'hui d'une bien vivante sociologie de la bureaucratie, équipée des outils conceptuels fournis par les théories modernes de l'organisation, du pouvoir, de l'information et de la décision. On se doute bien que des expériences fort importantes et fort passionnantes se déroulent actuellement dans certains secteurs de l'administration provinciale, mais il ne se trouve personne pour les observer scientifiquement. Vous me direz qu'on ne permettrait pas à des intrus de faire l'étude de tout cela, mais a-t-on seulement tenté l'aventure ?

Nous sommes encore plus pauvres, si c'est possible, en études sur les groupes de pression, alors qu'aux États-Unis, en France et en Angleterre ces études sont de plus en plus nombreuses. Dans ce domaine aussi l'observation n'est pas facile, mais la difficulté devrait être davantage un stimulant qu'un empêchement. Nous continuons de penser notre système politique en termes de pouvoir exécutif, de pouvoir législatif, et de pouvoir judiciaire, et dans ce schéma la bureaucratie, les groupes de pression, les opinions publiques s'intègrent mal ou pas du tout. De même, comme l'indiquait $\mathrm{M}$. Bonenfant, nous continuons de penser le fédéralisme canadien en des termes juridiques qui recouvrent mal ou pas du tout les phénomènes sociaux, économiques et 
même politiques qui se produisent réellement. Nous ne serons sauvés de ces mauvaises habitudes, qui se traduisent parfois en mauvaise politique, que par des théories nouvelles, partielles ou globales, qui rendront compte de tous les faits visés, en les expliquant les uns par les autres.

C'est aussi à un traitement par des méthodes nouvelles d'analyse du contenu qu'il faudrait soumettre nos idéologies politiques et leurs différents avatars, les considérant comme des mythes propres surtout à réduire des contradictions inscrites dans nos structures sociales. Mais une telle approche présuppose une connaissance très poussée des structures sociales et de la culture, aussi bien matérielle qu'immatérielle : elle dépend donc des progrès qui seront faits dans d'autres domaines.

Au terme de ce commentaire je n'ajouterai rien aux pertinentes remarques de $\mathrm{M}$. Bonenfant sur la stratégie de nos recherches. Je crois avec lui qu'un certain dirigisme est nécessaire, et qu'il faut, pour une plus grande richesse, concilier en nous les apports américain, anglais et français. J'ajouterai seulement que, déjà, des départs peuvent être pris, en séminaire, autour d'un professeur. J'en ai fait une première expérience, durant le semestre qui s'achève. Une quinzaine d'étudiants sont allés en éclaireurs dans diverses directions et ils ont rapporté de riches découvertes, dont quelques-unes furent signalées ici. Ces premières explorations ouvrent la voie à des enquêtes mieux orientées où nos quelques chercheurs pourront poser aux faits des questions plus précises.

Souhaitons enfin que ces quelques chercheurs se multiplient, non pas pour établir l'hégémonie de nos disciplines sur les autres, mais pour que notre contribution, qui est de montrer comment sont coordonnées les relations sociétales, ait sa place dans la connaissance plus exacte que nous aurons demain du Canada français.

Vincent Lemieux

Département de science politique, Université Laval.

Fin du texte 